aGp

Broadsheet No 126

October 1990

\title{
Examination of specimens of the larynx
}

\author{
L Michaels
}

Situated at the gateway to the lungs, the larynx affects profoundly the oxygen supply of the body; pathological changes are therefore always of great clinical concern. Pathologists are frequently called on to assess changes in the larynx both in surgical specimens and at necropsy. The use of a sound technique is vital to this investigation.

\section{Surgical specimens}

LARYNGEAL BIOPSY SPECIMENS

Following the successful use of the colposcope for observation of diseases of the cervix uteri, a microscopic procedure was introduced for direct laryngoscopy. Not only can the mucosa of the larynx be observed under magnification, but also operative manipulations, especially biopsy, can be carried out with precision. Microlaryngoscopy under general anaesthesia, with biopsy of any abnormal areas, is now the standard technique in most ear, nose, and throat clinics for the investigation of laryngeal diseases. An important factor in the success of laryngoscopic biopsy is the rapid and efficient handling of the specimen. The biopsy fragment is conveniently fixed immediately by washing the tip of the biopsy forceps in formaldehyde fixative as soon as they are removed. Specimens may also be required in a fresh, unfixed state for frozen section (see below), or microbiological culture.

\section{Frozen section}

Frozen sections for rapid diagnosis of laryngoscopy biopsy specimens should not normally be considered. There are, however, occasional indications for frozen section to be performed on laryngoscopy specimens.

(a) In some cases no histological diagnosis can be obtained after repeated direct laryngoscopic biopsies (each requiring a general anaesthetic) have been carried out. Such cases include carcinoma with overhanging areas of inflammation or oedema so that diagnostic tumour tissue cannot be reached. Under these circumstances a frozen section may be of help in guaranteeing that the biopsy has yielded some diagnostic material. (b) Special procedures may be required in a particular case, such as electron microscopy or microbiological culture. Frozen sections may be obtained so that an immediate decision can be made as to whether such special laboratory procedures should be carried out and the appropriate methods of preservation used.

THE PARTIAL LARYNGECTOMY SPECIMEN

When examining the partial laryngectomy specimen it is essential first to identify grossly the normal structures and the neoplasm within the resected specimen. The resection margins may be painted with India ink to assist in their identification in the histological section. It is convenient in these specimens to slice the specimen vertically. This is done sequentially with a large scalpel using a new disposable blade, which is usually sufficient to cut through the ossified thyroid cartilage, providing a series of slices through the cartilage and overlying mucosa. In each slice the same surface is sampled for section in the embedded specimen, conveniently, the lateral surface. This is ensured by marking the opposite surface with a daub of India ink before embedding.

Sections are assessed, as in the whole laryngectomy specimen (see below), for origin of

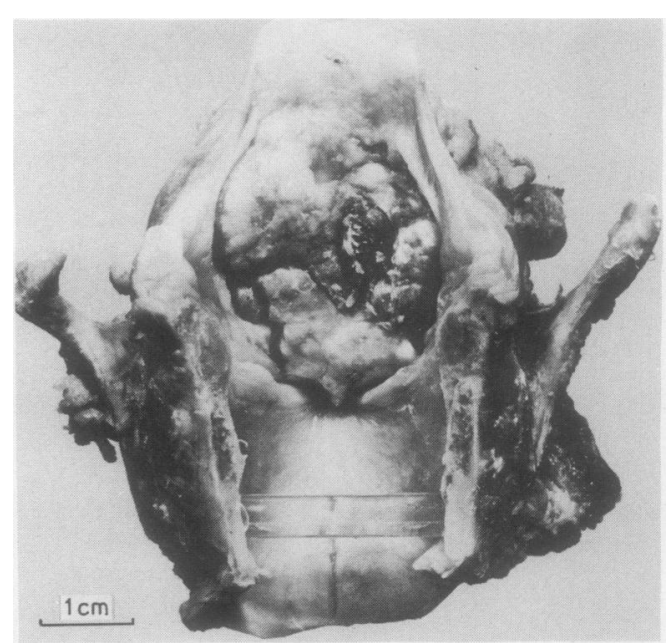

Figure 1 Larynx opened from behind to reveal a large supraglottic neoplasm. 
Figure 2 Motorised slicing machine for cutting fixed specimen of larynx.

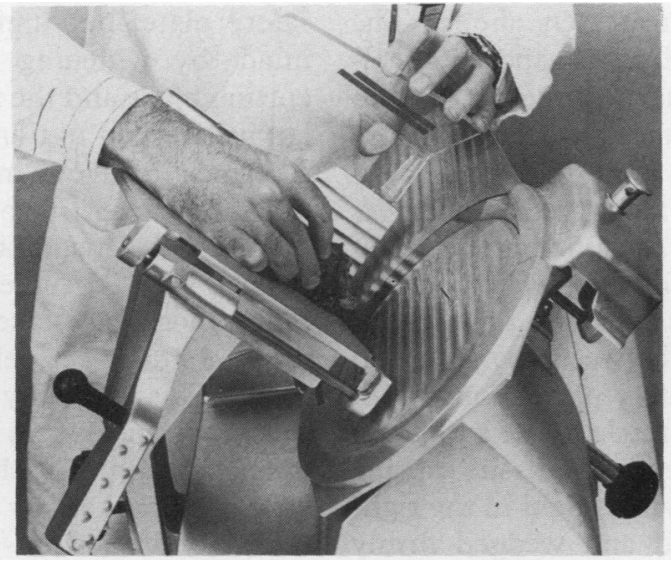

squamous carcinoma and extent of invasion, particular care being paid to the inspection of surgical resection margins for extension of neoplasm.

\section{LARYNGECTOMY SPECIMENS}

To obtain an adequate picture of the extent of spread of tumour in laryngectomy specimens multiple sections of the whole specimen are required. One means of achieving this has been the whole organ serial sectioning method. In this method the whole larynx is

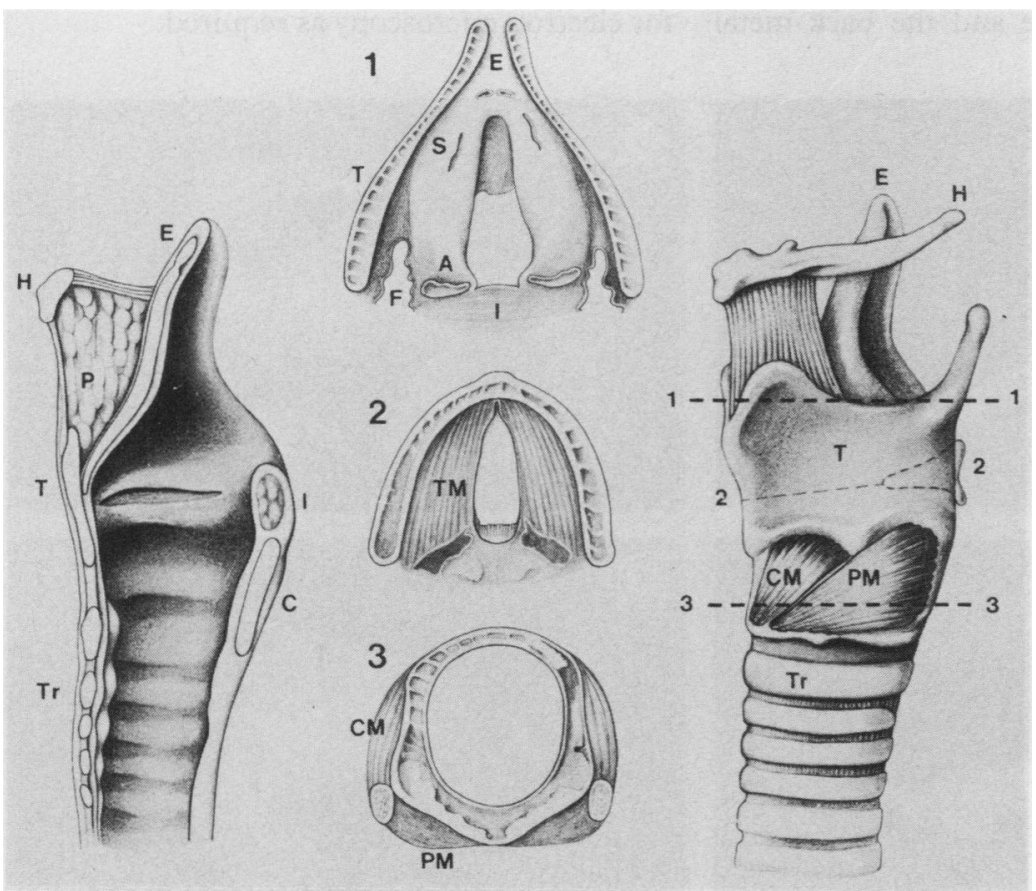

Figure 3 Diagrams indicating the structures seen in the intact normal larynx and on vertical and transverse slicing.

On the right, the whole larynx with the hyoid bone $(H)$ and epiglottis $(E)$ is viewed from the left side. The interrupted line (2-2) shows the position of the glottic transverse slice, 2, passing through the true vocal cords and arytenoids. The interrupted lines, 1-1 and 3-3, indicate the positions of the supraglottic and subglottic slices, 1 and 3, respectively.

On the left, the larynx has been bisected in a vertical plane and the right half is vieved from the left side. Note the hyoid bone $(H)$ and the epiglottic $(E)$, thyroid $(T)$, cricoid (C) and tracheal ( $\mathrm{Tr}$ ) cartilages in vertical section. Note also the fatty pre-epiglottic space $(P)$ and the interarytenoid muscle $(I)$ (the latter above the cricoid lamina $(C)$ ). Transverse slices, in the central row, are from the supraglottis, 1, glottis, 2 , and subglottis, 3 . The supraglottic slice, 1 , is bounded by the thyroid cartilages $(T)$ at the sides. The gap anteriorly represents the thyroid notch. Note the epiglottic cartilage (E) in transverse section. Behind it on each side is a slit-shaped saccule $(S)$. The apical portions of the arytenoids $(A)$ are seen posteriorly. Behind them and joining them is the interarytenoid muscle (I). At its two sides are the piriform fossae $(F)$ of the hypopharynx. The transverse slice through the vocal cords, 2 , showes the prominen thyroarytenoid muscles (TM) at this level. The lower slice, 3, is taken in the subglottis at the level of the cricoid ring. Note the cricothyroid muscles (CM) laterally and the posterior cricoarytenoid muscles (PM) posteriorly. ${ }^{4}$ cut serially after decalcification and embedding in celloidin ${ }^{1}$ or paraffin wax. ${ }^{2}$ The coronal plane is favoured for the serial sectioning of most laryngeal tumours. By contrast, serial sectioning of epiglottic tumours is performed in the sagittal plane.

Serial sectioning of the larynx is, however, far too time consuming for use in most histopathology laboratories. The method necessitates prior decalcification of the whole organ, a process that requires longer exposure to acid than is necessary for smaller blocks of tissue, and therefore leads to inferior histological appearances. In addition, with this method the opportunity for gross study of special areas is lost, and the application of modern histological methods, such as frozen sections, plastic embedding, immunocytochemistry and electron microscopy, is not possible. Whole organ serial sectioning requires long periods of embedding, which do not suit the clinical need for a reasonably quick laboratory assessment of the degree of tumour spread.

Using the method of Browning et al, ${ }^{3}$ three large vertical blocks of tissue were prepared by sagittal section through tumour and adjacent larynx. This method does not allow an adequate gross study to be made of the tumour in the larynx, particularly in the case of large or posteriorly situated tumours. The close proximity and interconnectedness of the thyroid alae, the cricoid lamina, and the arytenoids in this part of the larynx makes it difficult for well aligned slices of tissue to be cut by vertical section in this area. The need for whole organ decalcification again results in a histologically inferior preparation.

The introduction of computed tomography allowed a series of horizontal radiographs of the larynx to be taken at $5 \mathrm{~mm}$ intervals. To correlate the appearances of such radiographs with pathological changes, horizontal slices of larynges at similar intervals, using a slicing machine, were prepared. This method gave a complete gross picture of the tumour in situ in the larynx, and satisfactory histological studies were obtained of the material so sliced. ${ }^{4}$ The same method is recommended for routine examination of laryngectomy specimens. The blade of the slicing machine cuts smoothly through the ossified cartilages. Although possible in small fragments of ossified larynx (see above), such incisions cannot easily be made with a stout knife or scalpel on the whole larynx.

\section{Preparation technique}

The larynx is fixed in $10 \%$ buffered formolsaline for at least 48 hours. It is then opened by a vertical cut along the midline of the posterior surface. After the gross appearances of the interior of the larynx have been described (fig 1) the hyoid bone is carefully dissected off the larynx. If tumour is seen in the pre-epiglottic space, either grossly at this stage or microscopically at a later stage, the hyoid is sectioned transversely by sawing, and sampled for histological examination.

The larynx is then sliced transversely in a machine of the ordinary meat-slicing motor- 
ised type used by delicatessen shops. The machine is always supplied with special grindstone equipment for sharpening the circular blade. It is very important to do this before cutting each larynx so that the incision of ossified areas will be facilitated. Slicing is carried out transversely, starting at the tip of the epiglottis. The machine is set for cutting slices of $4 \mathrm{~mm}$ thickness by turning the wheel, regulating the distance between the spherical cutting blade and the safety plate. Four millimetres is the maximum thickness of a block of tissue that can be instited into a tissue capsule for embedding. The larynx is held in the right hand at its inferior end and wedged firmly against the vertical plate on the movable tray so that its posterior surface is downward. The tip of the epiglottis may be removed with scissors before the first slice. The spiked holder is applied to keep the specimen firmly in position. Slices are produced by sliding the movable tray sharply against the moving circular blade with the left hand (fig 2). When each slice is cut, it is carefully orientated so that it represents a view of the specimen as seen from above. A sequence of slices is easily produced in this way, displaying the whole larynx smoothly and evenly. Occasionally a section from a heavily ossified larynx may become wedged after cutting between the cutting blade and the back metal

Figure $4(A-F)$ Series of transverse slices obtained from the larynx bearing supraglottic tumour shown in fig 1 . The slices are placed serially from upper epiglottis region in $A$ to subglottis in $F$. The anterior edge of the tumour is demarcated by arrowheads. It is seen to be invading the epiglottic cartilage $(E)$ into the preepiglottic space in $B, C$, and D. It lies above and separate from the vocal cords (VC) in E. In slice $F$, at the level of the lower part of the true vocal cords, the normal cricoarytenoid joints may be identified (arrows). There is no tumour at this level. The tumour showed a glandular malignant structure on light microscopy, was neurone specific enolase positive on immunocytochemistry, and exhibited neurosecretory granules on electron microscopy, all of which led to a diagnosis of neuroendocrine carcinoma. ${ }^{4}$
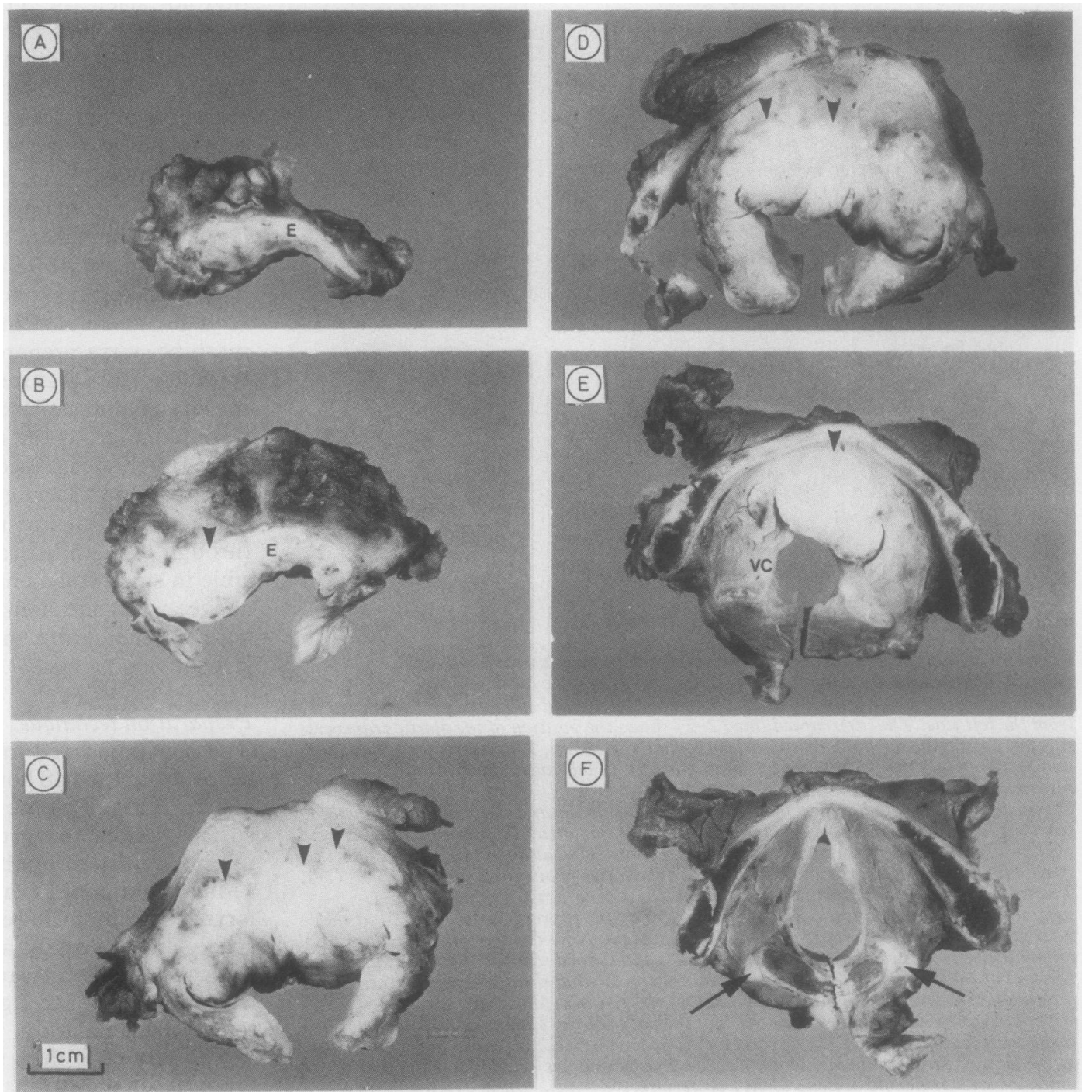

safety plate, but it may usually be removed intact by widening the space between the cutting blade and the safety plate. The slices of larynx are laid out and identified by letter in sequence. Each one may be photographed by a Polaroid camera to obtain a black and white picture at 1:1 magnification.

Each slice is studied grossly, and representative blocks are taken for histological study using a sturdy scalpel with a fresh disposable blade. This is usually sufficient to cut through the $4 \mathrm{~mm}$ thick slices of ossified cartilage. The exact position of each block taken for microscopy is marked by drawing corresponding lines with a black felt-tip pen on to the Polaroid photograph. The tissue blocks are carefully orientated after paraffin wax embedding by marking the reverse surface to that to be cut with India ink. The blocks taken for microscopy are decalcified, processed, and embedded in paraffin wax; sections are cut at $4 \mu \mathrm{m}$ and stained in the usual way. Each histological section is easily related to its origin on the tissue slice by placing it on to the corresponding Polaroid photograph (which is the same size as the original tissue slice) so that it fits into the shape made by the felt-tip pen lines. Selected areas of tissue may also be subjected to frozen sectioning, plastic embedding, or processing for electron microscopy as required.

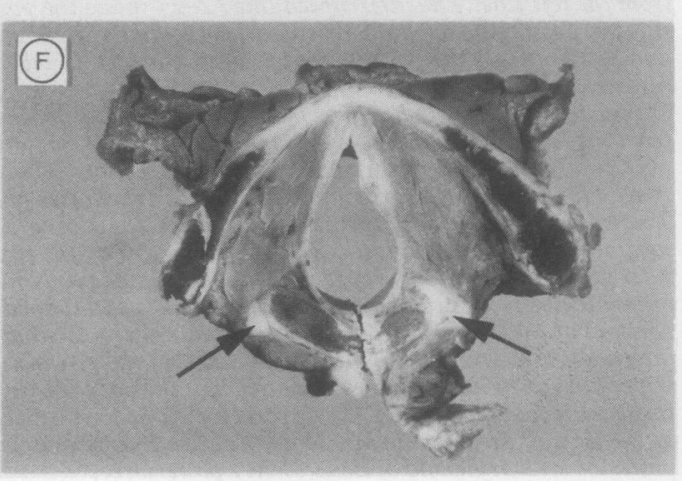


Figure 5 Horizontal slice of normal larynx seen at necropsy at the level of vocal cords and cricoarytenoid joints. The thyroarytenoid $(T)$, lateral cricoarytenoid (L), and posterior cricoarytenoid $(\boldsymbol{P})$ muscles can be identified.

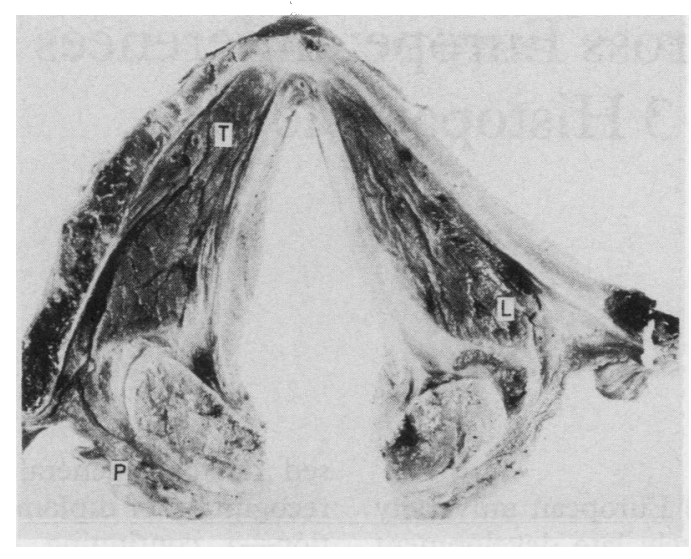

Appearances of transverse slices

The following normal structures may be identified in the tissue slices: epiglottis; laminae of the thyroid and cricoid cartilages; corniculate and cuneiform cartilages; false vocal cords; ventricles and saccules; true vocal cords; arytenoid cartilages; inferior cornua of the thyroid cartilage; intrinsic muscles; cricoarytenoid joints; cricothyroid membrane; and arch of the cricoid cartilage (fig 3). Any small structure that is not displayed on the surface of a block for microscopical examination, may be included within a paraffin wax block and can subsequently be displayed in histological section by cutting down on to the required area during microtomy. The full extent of the origin and invasion of the neoplasm in the larynx can be mapped out (fig 4).

Portions of hypopharynx that are removed with the larynx can be studied in the horizontal sections, and the method described is also suitable for investigating hypopharyngeal carcinoma that has been treated by pharyngolaryngectomy.

\section{Necropsy specimens}

THE LARYNX IN INFANTS

The small size of the infant larynx requires a different technique from that used for examining the adult larynx. The infant larynx is unossified. It may first be opened from behind for inspection of the lumen by a vertical incision before wax embedding. A sharp scalpel is sufficient to incise the specimen. The tip of the epiglottis may be removed for separate sectioning. The larynx is then cut horizontally into three separate slices, the superior one consisting of most of the supraglottis, the middle one containing the glottic region and adjacent supra- and subglottis, and the lower one the subglottis and upper part of trachea. The three slices are embedded and sectioned so that the sections on the slide represent the larynx as viewed from above. Step sections are cut through each block to obtain representative sections through the whole larynx.

\section{INTRINSIC LARYNGEAL MUSCLES}

This is usually carried out in necropsy specimens to assess pathological changes that might have damaged the neuromuscular pathways. A complete examination of this pathway would include the nucleus ambiguus, vagus, and recurrent laryngeal nerve and the intrinsic laryngeal muscles. ${ }^{56} \mathrm{~A}$ series of transverse slices taken in a similar manner by the technique used for the laryngectomy specimen exposes the intrinsic muscles of the larynx each of which may be sampled for histological examination (fig 5).

1 Tucker GF Jr. A histological method for the study of the spread of carcinoma within the larynx. Ann Otol Rhinol Laryngol 1961;70:910-21.

2 Tucker GF Jr. Human larynx coronal section atlas. Washington, DC: Armed Forces Institute of Pathology, 1971.

3 Browning GG, Bussutil A, McLay A. An improved method of reporting on laryngectomy specimens. J Pathol of reporting on

4 Michaels L, Gregor RT. Examination of the larynx in the histopathology laboratory. J Clin Pathol 1980;33:705-10. 5 Michaels L. Ear, nose and throat histopathology. London: Springer Verlag, 1987.

6 Quiney RE, Michaels L. Histopathology of vocal cord palsy from recurrent laryngeal nerve damage. J Otolaryngol 1990; (in press). 